\title{
Administrer la base de données : une fonction identifiée mais à pérenniser au musée national de Sèvres, Cité de la céramique
}

\section{Élise Fin-Osinski}

\section{(2) OpenEdition}

Édition électronique

URL : https://journals.openedition.org/ocim/1562

DOI : 10.4000/ocim.1562

ISSN : 2108-646X

Éditeur

OCIM

\section{Édition imprimée}

Date de publication : 1 septembre 2015

Pagination : 18-24

ISSN : 0994-1908

\section{Référence électronique}

Élise Fin-Osinski, « Administrer la base de données : une fonction identifiée mais à pérenniser au musée national de Sèvres, Cité de la céramique ", La Lettre de l'OCIM [En ligne], 161 | 2015, mis en ligne le 01 septembre 2016, consulté le 21 septembre 2021. URL : http://journals.openedition.org/ocim/ 1562 ; DOI : https://doi.org/10.4000/ocim.1562

Ce document a été généré automatiquement le 21 septembre 2021

Tous droits réservés 


\title{
Administrer la base de données : une fonction identifiée mais à pérenniser au musée national de Sèvres, Cité de la céramique
}

\author{
Élise Fin-Osinski
}

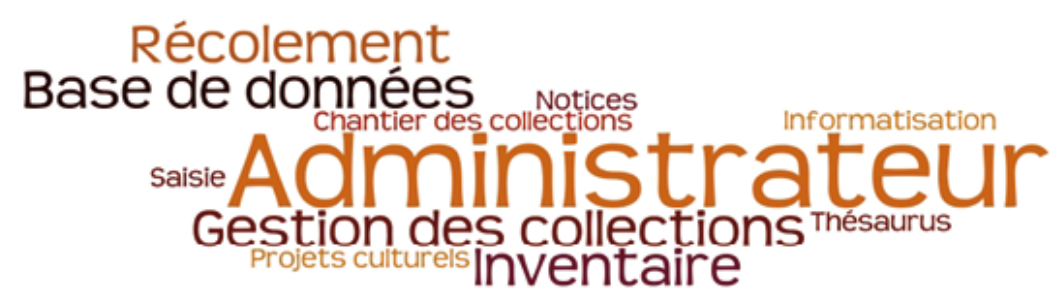

\section{(c) Élise Fin-Osinski}

1 Les questions relatives à l'informatisation des collections et à la gestion de base de données ne sont pas récentes. Toutefois, il est encore rare de voir ces missions dévolues à un professionnel spécifique au sein des institutions muséales, les fonctions d'administrateur de base de données étant bien souvent confiées à un conservateur, un chargé de collection, un chargé d'études documentaires ou un secrétaire de documentation dont le travail est déjà prenant.

2 Il y a quelques années, le travail à mener sur la base de gestion des collections du musée national de céramique de Sèvres était important. L'outil manquait de lisibilité et d'homogénéité. L'application semblait complexe pour les membres de l'équipe scientifique, alors restreinte, et présentait un intérêt limité. La prise de conscience progressive du potentiel de l'outil, au cœur de toutes les activités et projets de 
l'établissement, a amené l'institution à faire appel à un professionnel en charge de l'administration de la base. Cet article a pour objectif de partager l'expérience menée et les réflexions issues de la conception et de l'utilisation collectives de la base de données en montrant le rôle essentiel tenu par l'administrateur.

\section{L'obligation du récolement et l'apparition de nouveaux besoins}

3 Le musée national de céramique de Sèvres dispose d'un outil de gestion des collections depuis 1995. Après une première intégration d'environ 1500 notices, l'outil est longtemps resté inutilisé, du fait notamment d'habitudes de travail ancrées. La date butoir de juin 2014 pour la réalisation du récolement décennal approchant, de nouvelles procédures ont été mises en place en interne et il fut décidé de réaliser le récolement via le module spécifique de notre base informatique validé par le Service des musées de France. Cette nouvelle démarche pour le récolement est relativement récente, puisque celle-ci a véritablement débuté en 2010, mais symptomatique de l'ensemble des musées. L'année 2010 correspond également, à Sèvres, au renforcement de l\&apos ;équipe scientifique et donc à davantage de moyens humains pour effectuer le récolement des œuvres ${ }^{1}$. Ce sont les besoins liés à cette obligation qui ont permis d'impulser un nouvel intérêt pour la base de données.

\section{Quel profil pour l'administrateur ? À quelles limites est-il confronté ?}

4 L'expérience et le travail menés à Sèvres permettent de dessiner le profil de ce professionnel et d'évoquer certaines limites à son action.

5 L'administrateur de base de gestion des collections doit, avant tout, posséder des connaissances en recherches et normes documentaires. Il doit maitriser le système des thésaurus, de l'indexation, connaître les vocabulaires scientifiques, les terminologies...

6 À cette compétence documentaire s'ajoute une sensibilité pour le ou les domaines artistiques représentés dans les collections de l'institution où il travaille. Sans être forcément spécialiste du domaine en question, une formation en histoire de l'art ou des connaissances artistiques et historiques sont importantes pour appréhender l'objet, saisir les données relatives à celui-ci dans la base et, parfois, le manipuler.

7 De plus, l'administrateur doit avoir de solides connaissances du fonctionnement d'un musée, de la gestion administrative des collections et de la législation en vigueur (Inventaire règlementaire, récolement décennal, procédures d'acquisitions...).

8 Enfin, des connaissances en informatique et outils bureautiques sont indispensables pour assurer ses missions.

9 L'administrateur de base de données doit également posséder un certain nombre de qualités parmi lesquelles le sens de la pédagogie, l'écoute et la disponibilité afin de répondre aux demandes des utilisateurs et les assister. Il doit savoir s'adapter, travailler en équipe, faire preuve d'initiatives et être capable de coordonner des projets faisant intervenir la base de données. La rigueur et, plus encore, la capacité à concevoir et mettre 
en œuvre des méthodes adaptées, sont également obligatoires pour mener à bien son travail.

Le musée national de Sèvres, Cité de la céramique

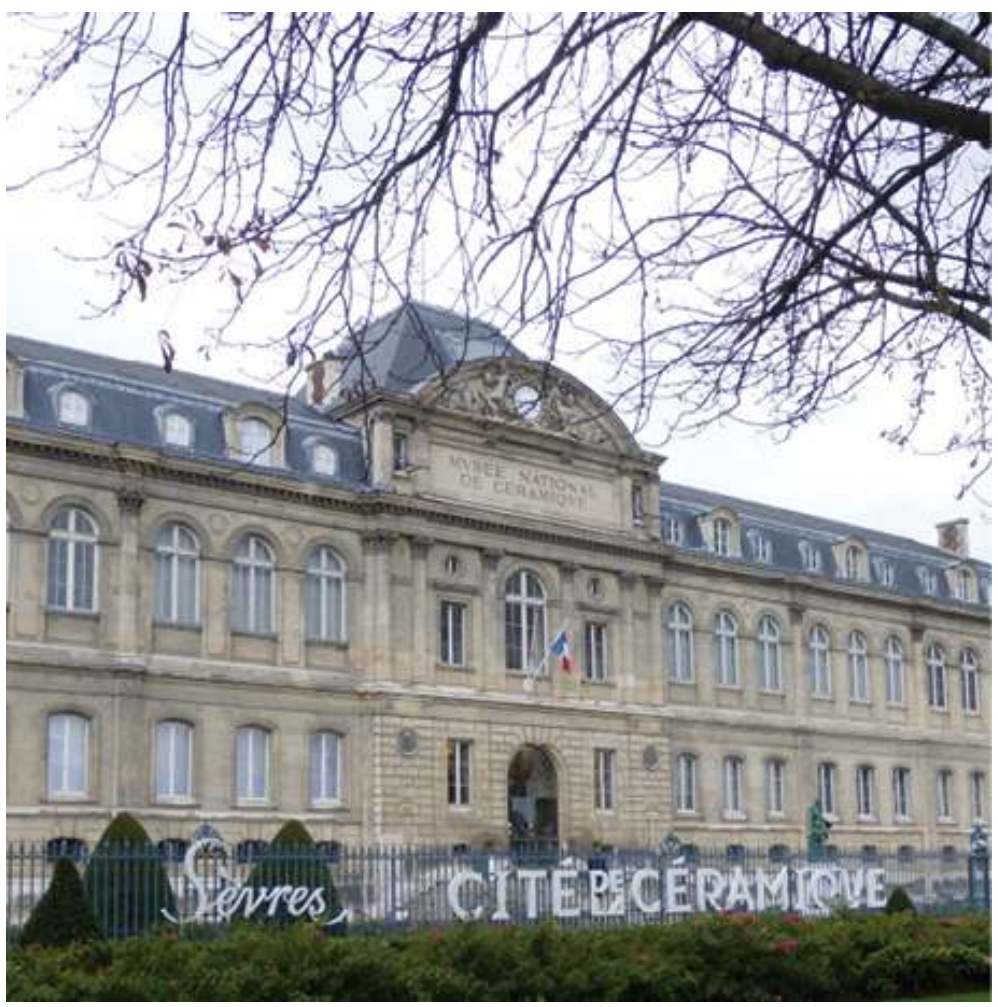

(c) Élise Fin-Osinski

D'autre part, les fonctions d'administrateur de base de gestion des collections se confrontent à certaines limites. En effet, jusqu'où ce dernier peut-il aller dans son intervention sur la base notamment au regard du travail scientifique du conservateur? Pour résoudre cette question et éviter tout conflit, il est important que l'administrateur soit un collaborateur intégré à l'équipe dont le travail est reconnu et préalablement défini en fonction des besoins et en accord avec le Projet Scientifique et Culturel de l'établissement.

11 Dans la mesure où la base de données est un outil collectif, il est nécessaire que tous les membres de l'équipe soient impliqués dans sa construction. «Si les corrections répétitives portant sur des ensembles cohérents relèvent de l'administration de la base, il importe que chacun corrige et mette à jour, ou signale les cas rencontrés susceptibles d'échapper à des recherches systématiques $\|^{2}$. Cette implication est parfois difficile à acquérir et à maintenir. La coordination de la base est un travail permanent qui doit recevoir l'approbation de tous.

Enfin, l'objectif de rendre la base de données toujours plus performante fait partie des premières intentions de l'administrateur. Toutefois, l'application ne cesse d'être perfectible. Ainsi, l'idée d'en faire un outil achevé est utopique. Ce n'est pas l'exhaustivité de l'informatisation qui doit orienter son travail et celui de l'équipe scientifique mais bien la réflexion sur un outil en évolution permanente. Concrètement, le travail de récolement a amené à définir une grille de saisie pour des notices succinctes. Une fois ce travail terminé, un nouvel objectif, celui d'un projet d'exposition, de mise en ligne ou d'indexation par exemple, entraîne la reprise des notices pour un nouveau type 
d'enrichissement. Ainsi les notices sont en perpétuel devenir ; il est donc nécessaire de cadrer les différentes actions pour obtenir une vision synthétique des collections à court terme, mais globale à moyen terme et enrichie à long terme.

\section{Quelles missions pour l'administrateur de base de données?}

Le traitement en masse des données est par nature un travail de longue haleine qui peut vite se révéler rébarbatif et déconcertant si l'on perd de vue les objectifs d'ensemble. Ce sont ces grandes lignes directives qui, malgré le travail de fourmi effectué sur les bases de données, permettent de donner un sens au travail de chacun, du stagiaire au décideur, et de valoriser les actions. Les missions de l'administrateur de la base s'inscrivent ainsi dans les projets et les stratégies du musée et, plus largement, de l'établissement. Cette partie s'attache à évoquer les principales missions confiées à l'administrateur au musée de Sèvres, des missions centrales qui témoignent de la reconnaissance nécessaire de cette fonction.

Le salon des grands vases.

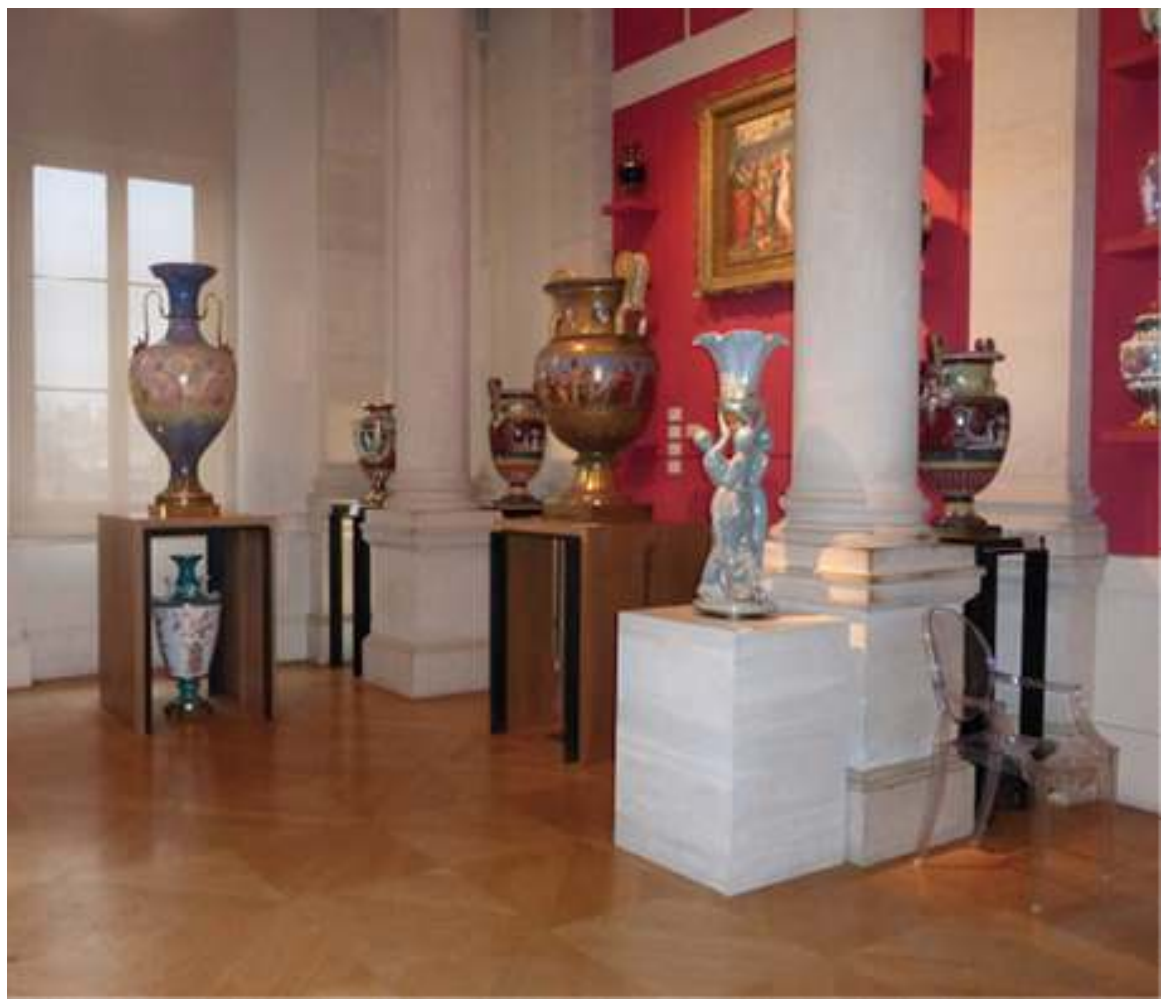

(c) Élise Fin-Osinski

\section{Une analyse pour permettre la reprise des données}

Après une première phase d'analyse de la base de données du musée national de céramique de Sèvres consistant en un bilan de l'existant - un bilan chiffré faisant apparaitre les thésaurus utilisés, la fréquence des termes, la qualité des données, l'état de la graphie ou encore la nature des erreurs présentes - une seconde phase de nettoyage et 
d'harmonisation a été entreprise. Tout d'abord, il s'agissait d'effectuer, entre autres, une fusion des doublons existants, des corrections, de mettre en place des normes (concernant la casse, la ponctuation, les abréviations, l'utilisation du pluriel...), de rationaliser les grilles de saisie... C'est-à-dire d'effectuer tout le travail préparatoire nécessaire pour obtenir un outil lisible et relativement propre permettant de mener une réflexion de fond. Puis, il s'est agi d'améliorer la qualité scientifique des données enregistrées, de construire et d'enrichir les thésaurus, ces listes regroupant des mots relatifs à un domaine de la connaissance, en mettant notamment en place des relations sémantiques entre les termes (hiérarchisation, synonymie, association). Cette structure de la pensée optimise les recherches documentaires dans le système informatique et facilite le travail scientifique à partir d'un outil collectif. Ce travail qualitatif de la base se poursuit au fur et à mesure des projets. En effet, l'inscription de tels objectifs au sein de projets scientifiques (exposition, chantiers d'œuvres, programmation...) facilite et structure leur mise en œuvre.

\section{La mise en place d'outils d'aide}

15 Pour une meilleure prise en main de l'équipe, des outils d'aide sont mis en place. Ceux-ci ont également pour objectif de rendre moins complexe la base de données et d'assurer $a$ minima son homogénéité en édictant des règles et des normes à appliquer par l'ensemble des utilisateurs. Ainsi, une charte de saisie a été rédigée et est mise à jour régulièrement. Également, le « lissage " progressif de la base amène des points de méthodologie diffusés sous la forme de fiches. Des procédures sont écrites pour faciliter certaines interventions sur l'outil, par exemple pour exporter des données ou importer des visuels. Ces documents sont enregistrés dans un dossier partagé sur le réseau permettant l'accès à l'ensemble des utilisateurs. Enfin, des documents de suivi sont aussi réalisés pour observer l'évolution de l'outil et expliciter certains choix opérés. En effet, les décisions prises doivent être consignées en vue de leur maintien sur le long terme afin d'assurer la cohérence du système.

La base informatique est par essence un outil commun qui doit prendre en compte les différentes collections conservées au musée et être un intermédiaire pour les différents projets. Ainsi, des séances de travail sont organisées afin de mener conjointement des actions visant à l'améliorer ou à l'adapter. Seule la priorité que l'on donne à l'outil en fait une véritable source d'informations qui en assure la pérennité.

\section{Le contrôle des données}

Qui dit base active, dit travail permanent. En effet, le flux constant d'informations relatives aux collections et le nombre d'intervenants sur l'outil (professionnels ou stagiaires) requièrent une grande vigilance et un nettoyage régulier. Les erreurs sont inhérentes à l'utilisation collective d'un tel outil amené à changer, à évoluer au fil des ans et des activités du musée. Même si un suivi du travail est effectué, tout ne peut pas être vu ni corrigé rapidement et la pollution de la base peut être rapide. D'autant plus que le travail de l'administrateur ne peut, dans les faits, se limiter à ce travail de contrôle et de vérification. En effet, d'une manière générale, tout projet ou question qui gravite autour des collections fait désormais intervenir la base de données. Ainsi, l'administrateur a toujours un rôle plus ou moins direct à jouer. 


\section{L'enrichissement de la base de données} l'informatisation des collections et les saisies ponctuelles pour répondre notamment à des demandes ou des besoins (expositions, demandes dans le cadre de recherches, demandes de prêt...). Cet enrichissement peut également passer par la récupération de données. Qu'il s'agisse de bases de données réalisées sous d'autres formats ou de campagnes de récolement non achevées, le problème reste celui des données non capitalisées. Dans ce cas, un travail de récupération de données et de traitement rétrospectif est parfois effectué en fonction de la priorité accordée au dossier et du temps disponible.

19 L'objectif est d'assurer une meilleure connaissance et visibilité des collections du musée, une étape scientifique primordiale vers la diffusion et la consultation par les publics.

\section{L'assistanat et la formation des utilisateurs}

20 L'administrateur reste disponible pour aider les utilisateurs dans la prise en main du logiciel et pour toutes questions relatives à l'enregistrement ou l'export de données. Parmi ses missions se trouve celle de former les futurs utilisateurs de la base (essentiellement des stagiaires). L'administrateur édicte les règles de saisie et veille à leur respect en éditant les outils d'aide évoqués précédemment.

21 Il s'agit là d'une mission essentielle pour pérenniser les bonnes pratiques et, a contrario, supprimer progressivement les mauvaises interventions afin d'assurer l'homogénéité et l'efficience de l'application. 
La salle consacrée à la porcelaine allemande des XVIII et $\mathrm{XIX}^{\mathrm{e}}$ siècles.

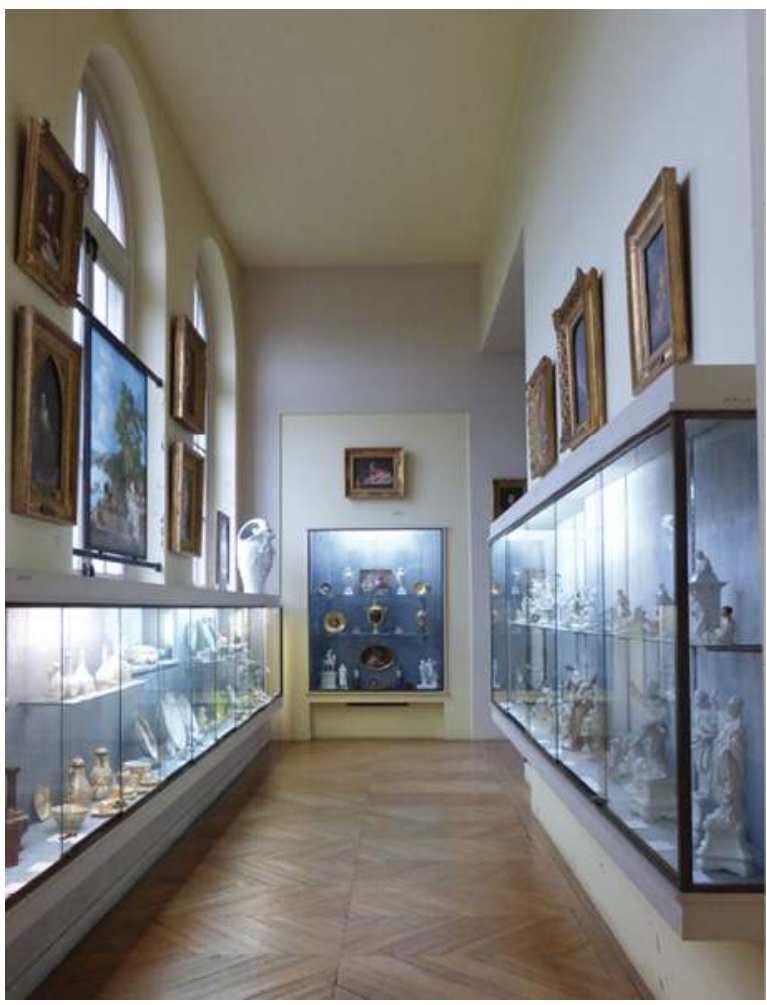

(c) Élise Fin-Osinski

\section{La gestion des droits d'accès, des paramètres et des prises de vue}

En accord avec l'équipe scientifique, l'administrateur définit des grilles de saisie différentes pour chacun des groupes utilisateurs (restrictions, champs obligatoires, rubriques non affichées...). Il s'agit d'une gestion adaptée au travail de chacun mais qui n'est pas pour autant figée. Celle-ci peut être réadaptée en fonction des retours d'expérience.

Une telle gestion réfléchie garantit la qualité et la pérennité des données sans pour autant empêcher l'utilisateur de travailler et permet de répondre aux objectifs qu'il s'est fixé. Ce paramétrage de la base assure également la traçabilité des données enregistrées qui peut se révéler utile en cas de recherches des interventions effectuées par chacun sur le logiciel.

D'autre part, l'administrateur veille au respect des normes et de la nomenclature définies pour les fichiers multimédias ainsi qu'aux poids et dimensions de ceux-ci avant intégration à la base de données. Cette gestion de la photothèque tend à limiter l'intégration d'erreurs lors d'imports en masse.

Une réflexion sur la capacité de stockage rend possible l'augmentation du nombre de visuels alimentant la base de données. De plus, les critères pour une photographie réussie ne sont pas à prendre à la légère. Leur respect fait de l'application un bon outil de travail ne nécessitant pas de retour sur les œuvres, et permet une diffusion satisfaisante des données. 


\section{L'adaptation de l'outil aux différents projets et la valorisation du travail de saisie}

L'administrateur est amené à répondre à certains besoins et à valoriser la base de données en la rendant utile et adaptable aux différents projets. En effectuant un catalogage des notices, en définissant une grille de saisie spécifique à un projet, en mettant en place une chaîne opératoire réutilisable (définition des objectifs, analyse de l'existant, rédaction d'une procédure, nettoyage et clarification dans la base, rédaction et diffusion d'une méthodologie à appliquer), l'administrateur fait de la base de données un instrument fiable de suivi de projets inscrits dans les missions ou les objectifs stratégiques du service des collections et de l'établissement (suivi de l'avancée du récolement décennal, de la couverture photographique dans le cadre du chantier de numérisation des œuvres...).

\section{Le récolement décennal des collections}

À Sèvres, la participation aux campagnes de récolement et l'encadrement de la saisie dans ces opérations font également partie des missions confiées à l'administrateur. Ce dernier édite les procès-verbaux et met à jour les chiffres de récolement. Il contribue ainsi à une meilleure connaissance des collections et s'assure que les données sur les pièces récolées soient enregistrées de manière uniforme afin d'avoir une idée générale de l'enrichissement de la base.

\section{Le suivi de l'activité sur la base}

Réaliser des statistiques de manière régulière permet d'avoir une vision générale de l'activité sur la base de données. Ces statistiques concernent le nombre de notices créées (entre 1995 et 2007, 12850 en 2008-2009, 36200 entre 2010 et 2014), le nombre de pièces récolées ou encore l'étendue de la couverture photographique. Il s'agit d'un travail préparatoire au rapport annuel fourni à la hiérarchie, mais aussi au bilan annuel de l'établissement. Au-delà de ce premier objectif concret, le bilan chiffré ainsi obtenu valorise le travail fait en coulisses mais qui pourtant alimente toutes les actions menées. De plus, les statistiques rendent possible une analyse qui permet l'évolution de l'outil informatique et son inscription dans de nouveaux projets.

\section{Les demandes extérieures}

Du fait de sa connaissance de la base, l'administrateur répond aux différentes demandes qui lui sont transmises en effectuant des recherches documentaires. Les notices des pièces concernées peuvent être complétées et les pièces recherchées en vue d'accueillir les chercheurs. Dans ce cas, une participation scientifique est toujours proposée; celle-ci sera cataloguée dans la base de données. De nouveau, ce travail permet d'enrichir l'application, d'avoir une meilleure connaissance sur certains lots d'objets et de valoriser les collections. 


\section{Les échanges avec le service informatique}

30 En plus de ses connaissances sur la base de données, l'administrateur doit avoir une vision globale concernant le fonctionnement du réseau et l'architecture informatique des ressources utilisées. Ainsi, il est en relation régulière avec le service informatique de l'établissement pour les questions liées aux problèmes de performance, de stockage, pour l'installation des mises à jour, la création de sessions informatiques, les sauvegardes des données enregistrées... Tout ceci fait l'objet d'échanges fréquents permettant à l'administrateur d'avoir une meilleure compréhension du développement de l'application et de relayer, au besoin, certaines informations. Cette collaboration permet aussi un suivi de la maintenance de la base (historique des problèmes rencontrés, des interventions effectuées, des restaurations...), essentiel pour les professionnels amenés à se succéder.

En fonction de l'institution, des effectifs et des besoins ressentis, d'autres missions peuvent être confiées à l'administrateur, comme par exemple la mise à jour du registre d'inventaire dans le cas où celui-ci est informatisé, la gestion des nouvelles acquisitions, le traitement des dossiers de dépôt... C'est lui qui est en charge de l'évolution de l'outil, de la rédaction du cahier des charges et du suivi de la migration des données en cas de changement d'outil.

Tendre vers une base exemplaire pour diffuser les collections

Nombreux sont les musées aujourd'hui dont les collections sont accessibles sur Internet; plus encore, de nombreux réseaux se sont créés ou émergent, bien souvent à l'initiative des professionnels des musées comme par exemple Musénor (Association des Conservateurs des Musées du Nord-Pas de Calais), Aliénor (Association Conseil des musées de Poitou-Charentes) ou encore les réseaux des Musées de Méditerranée, des Musées Basse-Normandie, des Musées en Roannais... Le regroupement n'est pas seulement géographique mais peut être thématique comme RéMut, le réseau des musées et collections techniques.

À l'heure actuelle, les collections du musée national de céramique de Sèvres sont très peu mises en ligne. La création d'un portail documentaire sur les collections et les arts du feu en général fait partie des stratégies de l'établissement public Sèvres, Cité de la céramique, et figure dans le PSC rédigé en 2013. Ce projet s'appuie notamment sur la base documentaire (actuellement sur Micromusée) dont la bonne gestion sera garante de sa réussite. En partenariat avec d'autres collections publiques de céramique en région (Nevers, Rouen, Marseille...), ce portail serait une référence en matière de céramique et pour l'ensemble des musées conservant des collections hétérogènes.

\section{Conséquences de la présence d'un administrateur à Sèvres}

Après deux années de travail, le bilan de la présence d'un administrateur de base de données au sein de l'équipe du musée est positif et a permis une meilleure connaissance des collections tout comme une meilleure gestion informatique et physique des œuvres. 
Le fait qu'une personne ressource soit en charge de la base, une personne à même de porter assistance et de répondre aux diverses questions, s'est révélé primordial pour modifier progressivement les appréhensions et les habitudes. Ainsi, dès les premiers mois de travail, l'équipe scientifique a pu apprécier les changements opérés sur la base, devenue rapidement opérationnelle. Le travail mené l'a encouragé à découvrir l'application ou à améliorer sa compréhension et son utilisation de celle-ci.

D'autre part, la présence d'un administrateur a permis d'augmenter de manière significative l'activité sur la base informatique du fait de son apport propre mais aussi du nouvel intérêt impulsé, et des nouveaux besoins.

Grâce à la reconnaissance de l'importance de l'outil et des fonctions d'administrateur de base de données, celle-ci est désormais la colonne vertébrale de la gestion, la valorisation et la diffusion prochaine sur Internet des collections de la Cité de la céramique, Sèvres et Limoges.

Les administrateurs de base de données dans les musées nationaux

Un certain nombre de musées nationaux reconnaissent l'importance des fonctions liées à l'administration des bases de données. La plupart du temps, celles-ci sont confiées aux professionnels de la documentation comme c'est notamment le cas au MuCEM qui emploie un chargé et un secrétaire de documentation. Au musée Rodin, au musée Picasso ou encore au musée d'Orsay, un chargé d'étude documentaire remplit ces fonctions. Au musée de l'Armée, un poste consacré à l'administration de la base de données à été créé en 2014 pour décharger les documentalistes, chargés de récolement, mais plus encore, pour acquérir de nouvelles compétences au sein de l'équipe, afin d'engager des projets fondamentaux comme l'évolution de l'outil et la mise en ligne des collections. Dans d'autres cas, l'administration de la base reste le fait de conservateurs (au musée de Cluny ou au musée national des châteaux de Malmaison et Bois-Préau), ce qui était le cas à Sèvres jusqu'à récemment.

En fonction de l'établissement, de son historique et de ses collections, mais aussi de l'état de la base informatique, les besoins diffèrent. Ainsi, une petite structure ne ressentira pas la même nécessité d'y consacrer un poste. Au musée de l'Orangerie, dont les collections comprennent 154 œuvres, un fonds d'archives de 1350 pièces et 3000 dossiers documentaires, la base Videomuseum est alimentée par un chargé d'étude documentaire et un secrétaire de documentation, ainsi que par le régisseur pour la partie logistique ; rattaché depuis 2010 au musée d'Orsay au sein d'un même Établissement public, le musée de l'Orangerie a peu à peu basculé à l'été 2015 dans une nouvelle base commune appelée M'OSAIC et bénéficié de la présence de l'administrateur de base de données du musée d'Orsay. Le travail collectif sur ces questions s'avère en effet parfois suffisant. Ainsi, au musée national de la Renaissance d'Écouen, un conservateur, un documentaliste et un régisseur ont chacun la casquette d'administrateur et y consacrent du temps.

Et dans les musées territoriaux ? L'enquête est à mener. Toutefois, en leur donnant l'importance qu'elles méritent et en pérennisant ces emplois (n'oublions pas que récolement décennal notamment, est un travail continu), les fonctions de l'administrateur s'inscrivent plus largement au cœur du métier de Gestionnaire des collections (conservateur ou attaché territorial de conservation). 


\section{Pour conclure}

Il est fréquent que la base de données soit l'apanage des services de conservation. Toutefois, il faut parvenir à dépasser ce principe et faire de la base un outil vivant à l'image de l'établissement et porteur des différents projets. À Sèvres, l'outil reste certes la priorité du service des collections. Toutefois, avec le soutien de la hiérarchie, l'objectif est d'en faire un instrument de travail transversal, utilisé et enrichi par l'ensemble des services du Département du patrimoine et des collections de Sèvres, Cité de la céramique (services de la conservation préventive et de la restauration, du récolement et du mouvement des œuvres, des collections documentaires) selon les spécificités de chacun. On s'aperçoit que, bien souvent, les outils de gestion sont utilisés bien en deçà de leur potentiel.

L'expérience à Sèvres prouve que les fonctions d'administrateur de base de gestion des collections présentent différentes facettes qui nécessitent des compétences propres. Grâce à son expertise de la base, à sa vision d'ensemble sur l'avancée de l'informatisation, du récolement, de l'inventaire, à sa connaissance de l'architecture et de l'historique de la base, ce professionnel facilite le travail des équipes et se place ainsi au cœur des activités de l'établissement.

Dès 1995, Danièle Brochu précisait que "l'utilisation de l'informatique dans le secteur des collections faisait appel à des compétences souvent absentes du musée. C'est ainsi qu'un nouveau type de professionnel est apparu pour s'acquitter de ces nouvelles tâches $»^{3}$. Vingt ans après ces propos, il est encore trop rare de voir une personne reconnue chargée de ces missions de gestion de base de données, des fonctions qui restent un peu cachées et qui mériteraient de prendre davantage d'ampleur dans le milieu muséal français. D'autant plus que, malgré l'importance de ces missions et leur inscription au sein des projets d'établissement, les postes consacrés à l'administration des bases restent trop souvent précaires (contractuel, vacataire, prestataire), des statuts qui fragilisent les services et les établissements.

\section{BIBLIOGRAPHIE}

Arminjon, C. et Blondel, N. Objets civils domestiques - Vocabulaire typologique. Inventaire général des monuments et des richesses artistiques de France. Paris : Éditions du Patrimoine, 1984.

Blondel, N. Céramique - Vocabulaire technique. Inventaire général des monuments et des richesses artistiques de France. Paris : Éditions du Patrimoine, 2001.

Brochu, D. Manuel pratique d'informatisation : des collections à la base de données. Paris : Éditions d'art Somogy, 2004.

Fin, E. Administrateur de base de données : un métier au cœur de l'activité muséale. Mémoire de master professionnel sous la direction de Marc Andler et de Laurence Tilliard, UVSQ, 2012. 
Hudon, M., Dégez, D. et Ménillet, D. Guide pratique pour l\&apos ;élaboration d\&apos ;un thésaurus documentaire. Montréal : Les Éditions Asted, 2009.

Méthode d'inventaire informatique des objets : beaux-arts et arts décoratifs. Paris, Direction des musées de France, bureau de 1\&apos ;informatique et de la recherche, 1995.

www.culture.gouv.fr/documentation/joconde/fr/pres.htm

http://portail-joconde.over-blog.com

et plus particulièrement :

- Conduire un projet d'informatisation des collections : www.culture.gouv.fr/documentation/ joconde/fr/partenaires/AIDEMUSEES/projet.htm

- Fiche pratique sur la fonction d\&apos ; administrateur de base de données au sein d\&apos ;un musée : www.culture.gouv.fr/documentation/joconde/fr/partenaires/AIDEMUSEES/ administrateur.htm

- Siret, A.-S. Charte de saisie Micromusée : documents graphiques, peintures, sculptures, manuscrits. Collections des Beaux-arts de Paris, ENSBA, janvier 2014 : www.beauxartsparis.fr/ow2/catzarts/ doc/CharteSaisieMicrom.pdf

\section{NOTES}

1. Le taux de récolement était de $29 \%$ en juin 2014.

2. Siret, A.-S. Charte de saisie Micromusée, version 17. ENSBA, janvier 2014.

3. Brochu, D. Manuel d'informatisation des collections, des collections à la base de données, p. 13.

\section{RÉSUMÉS}

À partir de son expérience professionnelle, l'auteur montre comment la fonction d'administratrice de base de données des collections est peu à peu devenue un métier à part entière, reconnu pour son expertise et transversal au sein de l'institution muséale et qui requiert des compétences multiples ainsi que des qualités parmi lesquelles le sens de la pédagogie, l'écoute et la disponibilité.

\section{INDEX}

Mots-clés : base de données, collection, musée national de Sèvres, cité de la céramique 


\section{AUTEUR}

\section{ÉLISE FIN-OSINSKI}

attachée territoriale de conservation du patrimoine et administratrice de la base Micromusée au musée national de Sèvres, Cité de la céramique

elise.fin@laposte.net 